Engineering and Design

Elsevier Editorial System(tm) for Fusion

Manuscript Draft

Manuscript Number:

Title: DC Inculation Tests of W7-X Coils at CEA, Saclay

Article Type: Soft 25th Special Issue Paper

Keywords: DC Insulation test, test at cryogenic temperature; test facility, power supply, data acquisition system.

Corresponding Author: Mr Giancarlo Croari,

Corresponding Author's Institution: Max-Planck-Institut für Plasmaphysik

First Author: Giancarlo Croari

Order of Authors: Giancarlo Croari; Holger Viebke; Kerstan Hertel; Juergen Baldzuhn; Laurent Genini; Bertrand Renard; Laurence Vieillard

Abstract: The Wendelstein 7-X (W7-X) is an advanced optimized stellarator under construction at the Greifswald branch of the Max-Planck-Institut f.ur

Plasmaphysik. Its magnet system consists of 70 superconducting coils, i.e. five types of 10 non planar (NPC) and two types of 10 planar (PC) individuals each. In case of an emergency shut-down like in succession of a quench, the voltage across a NPC may raise up to 450 Volt according to the specification. Since all the 10 coils of a type are connected in series, the total voltage could thus reach $4.5 \mathrm{kV}$. Therefore, the coils had to be provided with a corresponding electrical insulation. Its test voltage levels were fixed at $10.4 \mathrm{kV}$ and $7.2 \mathrm{kV}$, respectively, for the NPCs and PCs.

The coil acceptance tests take place in an ad hoc facility at CEA-Saclay where a large variety of trials are performed, including the verification of the electric insulation quality. Several DC high voltage tests (DHVT) are carried out to assess the proper manufacture of the ground insulation which stretches across the winding pack, conductor terminals, interlayer joints, and the numerous quench detection wires including their connections. The DHVTs are performed under different conditions: before cooldown at room temperature and atmospheric pressure, then at cryogenic temperature and, finally, under vacuum at room temperature. Since the coils are necessarily connected to the test facility, the corresponding components like wires, plugs, or connections are also energized by the nominal voltage.

Thus a flaw in the external test bed may invalidate the outcome of the experiment. It is therefore very important to determine whether the origin of a current leak is in the coil or in the test facility.

To do so, it is necessary to have deep knowledge of the whole arrangement. The paper summarizes the experimental observations, data analysis, and consequent improvements which were implemented at CEA test facility. 


\title{
DC Insulation Tests of W7-X coils at CEA, Saclay
}

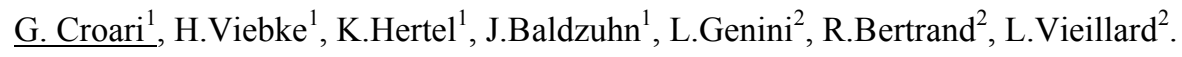

(1) Max-Planck-Institut für Plasmaphysik (IPP), Greifswald Branch, Euratom Association, Wendelsteinstraße 1, D-17491 Greifswald

(2) Commissariat Energie atomique, Saclay-Gif sur Yvette, F-91400.

Author's contacts: $\quad$ CEA, Saclay (F-91400) Tel.: +33 169085706

IPP, Greifswald (Gemany) Tel.: +49 383488 1844;

E-mail address: giancarlo.croari@ipp.mpg.de

\begin{abstract}
The Wendelstein 7-X (W7-X) is an advanced optimized stellarator under construction at the Greifswald branch of the Max-Planck-Institut f ur Plasmaphysik. Its magnet system consists of 70 superconducting coils, i.e. five types of 10 non planar (NPC) and two types of 10 planar (PC) individuals each. In case of an emergency shutdown like in succession of a quench, the voltage across a NPC may raise up to 450 Volt according to the specification. Since all the 10 coils of a type are connected in series, the total voltage could thus reach $4.5 \mathrm{kV}$. Therefore, the coils had to be provided with a corresponding electrical insulation. Its test voltage levels were fixed at $10.4 \mathrm{kV}$ and $7.2 \mathrm{kV}$, respectively, for the NPCs and PCs.

The coil acceptance tests take place in an ad hoc facility at CEA-Saclay where a large variety of trials are performed, including the verification of the electric insulation quality. Several DC high voltage tests (DHVT) are carried out to assess the proper manufacture of the ground insulation which stretches across the winding pack, conductor terminals, interlayer joints, and the numerous quench detection wires including their connections. The DHVTs are performed under different conditions: before cooldown at room temperature and atmospheric pressure, then at cryogenic temperature and, finally, under vacuum at room temperature. Since the coils are necessarily connected to the test facility, the corresponding components like wires, plugs, or connections are also energized by the nominal voltage.

Thus a flaw in the external test bed may invalidate the outcome of the experiment. It is therefore very important to determine whether the origin of a current leak is in the coil or in the test facility.

To do so, it is necessary to have deep knowledge of the whole arrangement. The paper summarizes the experimental observations, data analysis, and consequent improvements which were implemented at CEA test facility.
\end{abstract}

\section{1) Introduction: The aim of the electrical insulation test for the $W 7-X$ coils.}

The magnetic field of a W7-X coil is produced by its winding pack which is realized by different numbers of cable in conduit (CICC) turns. For the family of NPCs 108 turns are grouped in 6 double layers (DLs); whereas for a PC winding pack 36 turns are divided in 3 DLs. 
These CICC winding packs are wound from superconducting NbTi. Their dry insulation wrapped around the conductor aluminium jacket is better adapted to high voltage insulation than bath cooled conductors, more sensitive to short circuits [1].

The picture below shows the cross section of a NPC with a detailed zoom of the general insulation between the winding pack and the coil casing [2].

Figure 1: Cross section of a NPC and its detail of the Embedding insulation.

Aim of the DHVT is to prove the general insulation of a coil including its embedding insulation. These High voltage DC tests have as main goal to prove there is no current leak into the coil embedding insulation.

To test the inter-turn insulation, and therefore to detect a short circuit in the winding pack, other trials are required like $\mathrm{AC}$ test and impedance measurements.

\section{2) The choice of the test voltage.}

All the 10 coils of the same type will be connected in W7-X in series. Therefore 7 independent power supplies are required as well as the same number of Nickel discharge resistors [3].

By means of a fast discharge for one coil alone which undergoes the current test in the CEA test facility, a voltage value of roughly $400 \div 450$ Volt for NPC and $320 \div 360$ for PC are reached. These values are obtained in the self field since the coils are energized for testing one by one. In the past the table below was drawn to list the different voltage levels which were chosen based on the temperature and on the coil family [4].

Table 1: Test voltages for NPCs and PCs at room and cryo temperature.

Indeed the applied voltage increases with a 100 seconds ramp up to the indicated levels, then these levels are kept for 60 seconds and finally a ramp down to 0 has 100 seconds duration as well. The specified insulation resistance during the HV-DC test is $1 \mathrm{GOhm}$ per coil respective $500 \mathrm{Mohm}$ for an installation of two coils.

\section{3) The DC test equipment.}

The necessary equipment to perform a DC test is based on a Windows - Labview computer desk which is connected via GPIB to a PXI-National Instruments measurement device. This last object is then connected to a dielectric meter (or 'high voltage power supply') ELABO which has a current limitation of $400 \mu \mathrm{A}$ [5]. The Labview code for the control system and the user interface were developed by the informatics division SIS of CEA, Saclay.

At present status, this system is capable to provide a voltage (ramp up, plateau and ramp down), to display the voltage and current behaviours and to store a summary table in an electronic word file as a test report, but it doesn't store all the current and voltage samples.

\section{4) Components under high voltage DC test:}

Once the two coils are installed inside the vacuum tank of the cryostat, several components are connected with them. This list includes: as above mentioned the coil winding-pack insulation, the high-current connecting joints, 
potential breakers, wiring system for the quench detection system (QD-Wires), the current-leads (the current transitions from warm to cold of the test facility), and the bus-bar system. This complexity makes it difficult to localise a current leak, for the case that a high-voltage break-through occurs.

\section{a) On the coil.}

The components of a coil which are put under HV are the following:

- Embedding insulation:

As it was shown in figure 1 the hollow space between the coil casing and winding pack is filled with epoxy and has to prevent any movement of the winding pack into the casing.

- Insulation of the high-current Interlayer joints:

Two adjacent layers of the winding pack are connected by the interlayer-joints; the insulation with respect to the outer part is achieved by epoxy impregnated fibreglass.

- Potential breakers:

A potential breaker can be responsible of an insulation failure as well as a Helium leak. There is 1 potential breaker for each interlayer joint.

- QD wires:

These wires bring the interlayer voltages to the external data acquisition system. Their insulation is made of Kapton and they are externally ground shielded.

Figure 2: Header area of a type 4 NPC.

\section{b) On the test facility.}

In the picture below a top view of cryostat 2 with its relative valve box is shown.

Figure 3: A top view of cryostat 2 and its valve box.

The list of components of the test facility which are also energized by a DC test is the following:

- Vacuum feed-through for the Quench Detection Wires (QD-buckets):

These elements are screwed on the cryostat wall from the inner side and are necessary to allow a connection outside to the data acquisition system. One QD-bucket contains 6 plugs which are all energized since they are connected to the QD-Wires.

Figure 4: A QD bucket screwed on the lower part of the cryostat wall.

- Current leads:

The currents leads are contained in a so called "valve box". The outer part of the cryostat and of the valve box system is connected to ground, whereas the 3 current leads are energized with the high voltage (+ pole)

- Helium bath in the valve box:

The inner part of a valve box is kept under vacuum and each of the lowest parts of the 3 current leads is submitted to a Helium bath. 
Helium vaporization as well as a vacuum reduction may induce a voltage breakthrough by Paschen effect [6].

Figure 5: 3 tanks for Helium bath of the currents leads feet in the valve box volume.

- Joint boxes and bus-bars system:

Since these components are in strict contact with the coil terminals they are energized too. To prevent any discharge versus the cryostat wall, the extra length busses and the coil terminals are covered with a Kapton adhesive tape.

\section{5) Defects found on the coil by means of DC test.}

During the last 5 years some defects on the coils came out by DHVTs. For instance, on a type 5 coil in 2005 a void was revealed after several failures by DC test (picture on the left).

By inserting a camera into the cryostat it was possible to observe some flashes coming from the QD wire outlet on the header area of another coil type 2 . These coils were reworked by the manufacturer.

Figure 6: On the left a void in the connection area of AAB25; on the right a sparkling QD cable On AAB59.

\section{6) Improvements adopted on the test facility.}

Beside some imperfections of the insulation quality were discovered, some defects were suspected and investigated on the test facility too.

An improvement which was developed is the observation of the QD-bucket's plugs. A small tank is used to perform the DC tests of one QD-bucket under vacuum down to $10^{-4} \div 10^{-5}$ millibar. The faulty plugs can be easily detected by attempting the plugs one by one. It is a general rule to test each QD-bucket every 2 current tests. Another check point which was added is to test the cryostat alone under vacuum every two cold cycles.

Nowadays the ground insulation of the QD-buckets is much improved by means of additional insulation rings which are screwed between the cryostat wall and the QD-bucket flange. Thus a failure in a plug cannot any longer induce a current leak.

\section{7) General overview of the test results.}

Normally when a coil is in the hall the DC test is $100 \%$ successful; what makes things more difficult is when the coil is enclosed into cryostat and the DHVT either at cryogenic temperature or after warm up are performed. An IPP statement is that DC test after warm up is held only in case of failure at cold condition. The following table summarizes all the DHVTs when the coils are into the cryostat.

Table 2: Summary of all the DHVT at cryogenic temperature only. The reported numbers represent the number of tested coils. The improvement taken by the installation of the QD-rings can be observed by the graph below, the last update was done on 30/07/08. 
Graph 1: For NPCs the successful DC tests increased from $44 \%$ to $67 \%$; similarly for PCs the success rate passed from $53 \%$ to $60 \%$. *=one only planar coil at cold condition gave failed unlike at warm condition.

\section{8) Conclusion.}

The lesson learnt with the experience of W7-X coil testing at CEA is that it is quite challenging to localize a current leak in a system "coils + test facility", in case such leaks are present. In fact with the use of a camera into the cryostat some flashes were revealed on a certain coil, or by using a dedicated tank for QD-bucket test under vacuum a few plugs were discovered with poor quality insulation. The use of Teflon rings could improve the general average of the success but not yet bring the percentage to $100 \%$.

This proves the idea that investigating the responsibilities of a DC test failure can be a tough job.

What could be wishful for the next test facilities development is to get either an improvement of all the insulations for the components under HV or a reduction of the voltage levels.

A data saving system with an off-line analyser could probably be helpful to determine whether the current leak is on the test equipment or on the coil(s).

\section{9) Bibliography.}

[1] Bertrand Renard: W7-X Superconducting Coils Cooling at the CEA Saclay Cryomagnetic Test Facility SOFT 2008.

[2] Hartmut Emler: Ringbuch: Nichtplanare Spulen (KKS 1-AAB -R0001.1).

[3] Th. Mönnich , Th. Rummel: Production and Tests of the Discharge Resistors for Wendelstein 7-X.

[4] Scope and Requirements for the Acceptance Tests on the W7-X - Coils.

[5] Thierry Schild, Laurent Genini: Procedure de test d'une bobine non-planaire.

[6] Hartmut Ehmler: Electrical tests on W7-X Coils (1-AAF-Y002.0) 

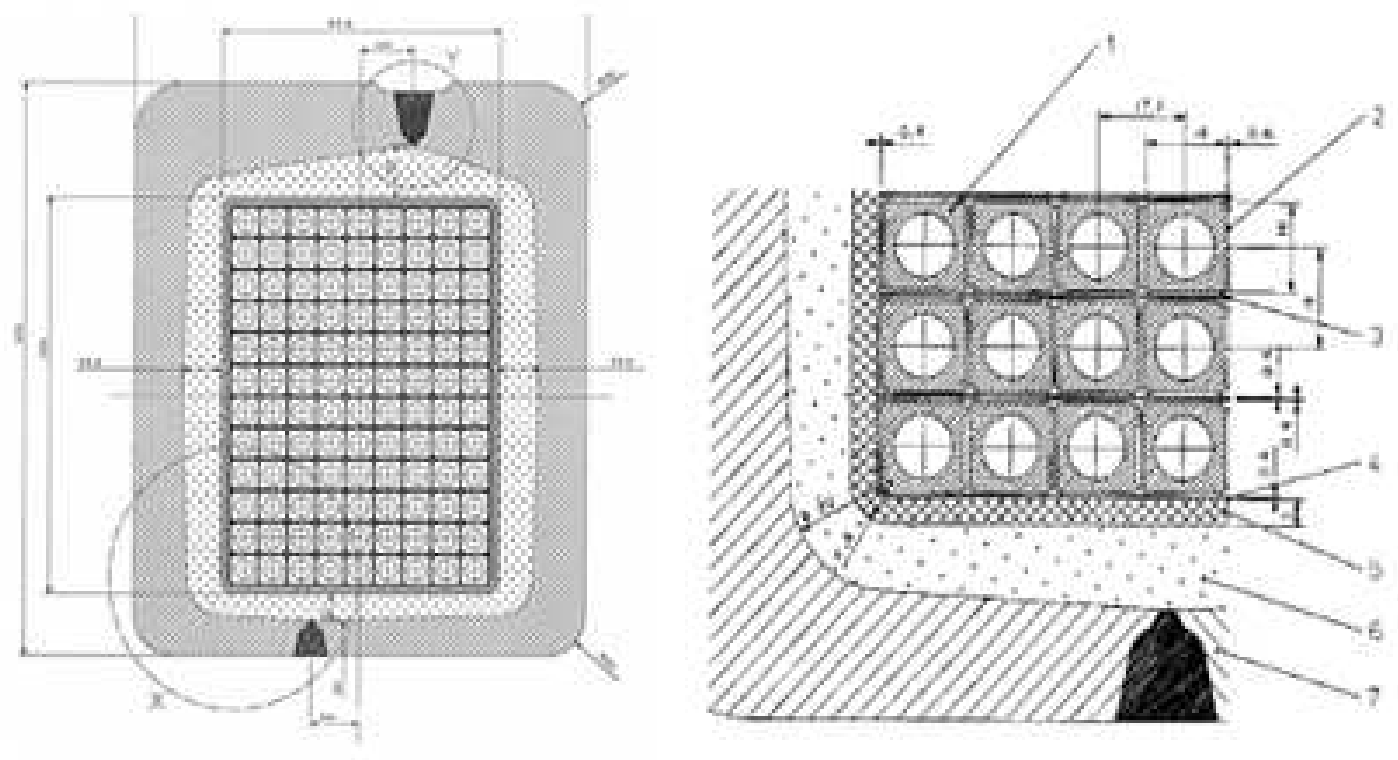

1. AL-Jadket

2. Speronductor Ireultion

3. Inter tum houlation

4. Irter tum Iosulation

5. Goond houlation

6. Embediting Frsultion

7. Coil cosing 


\begin{tabular}{|l|l|l|}
\hline & \multicolumn{2}{|c|}{ Applied Voltage } \\
\hline Temperature & Non-planar coils & Planar coils \\
\hline Room temperature & $9.1 \mathrm{kV}, \mathrm{DC}, 1 \mathrm{~min}$ & $6.3 \mathrm{kV}, \mathrm{DC}, 1 \mathrm{~min}$ \\
\hline Cryogenic Temperature (4 K) & $10.4 \mathrm{kV}, \mathrm{DC}, 1 \mathrm{~min}$ & $7.2 \mathrm{kV}, \mathrm{DC}, 1 \mathrm{~min}$ \\
\hline
\end{tabular}

Table 1: Test voltages for NPCs and PCs at room and cryo temperature. 


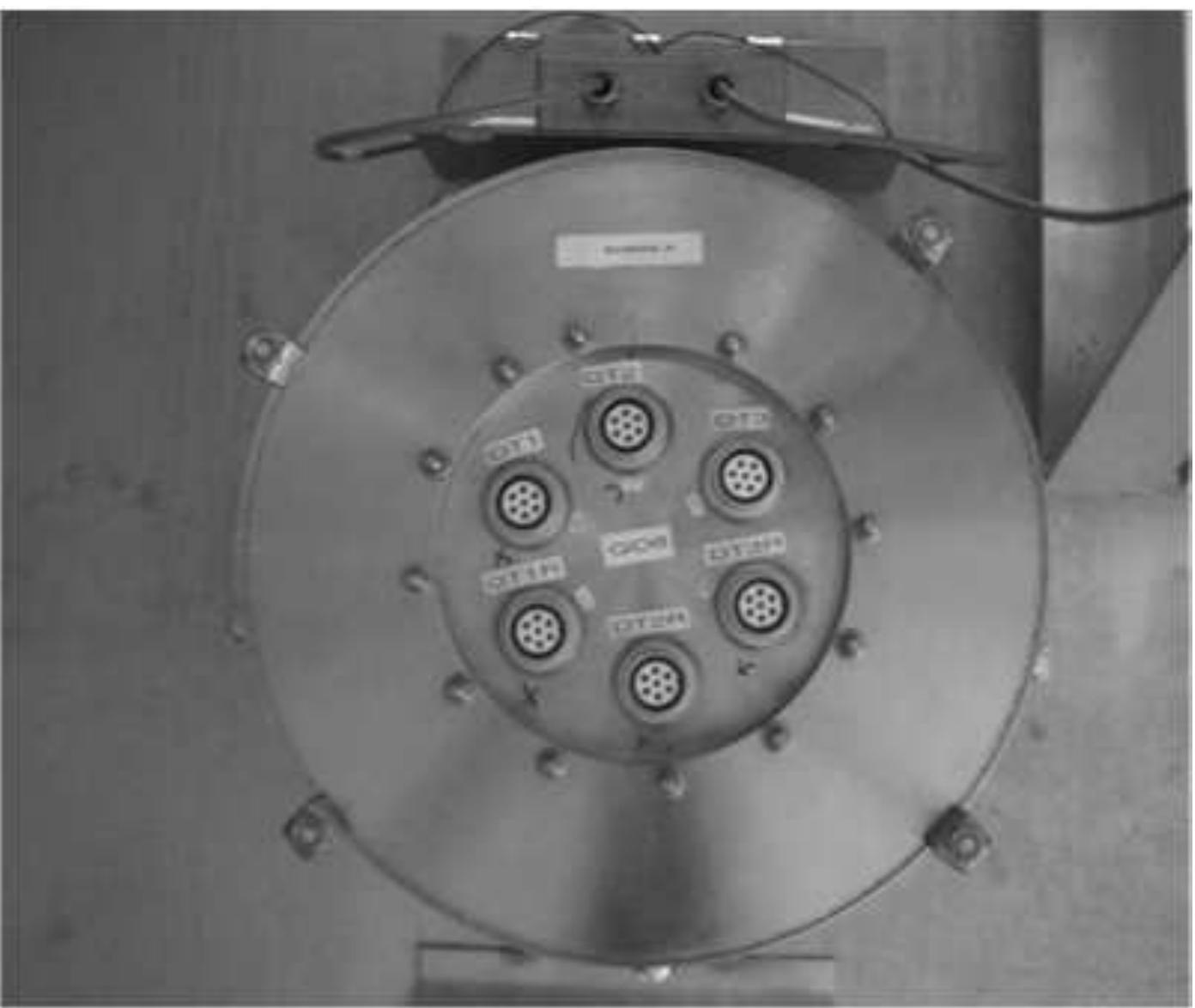

Figure

4

\section{Figure4}

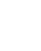

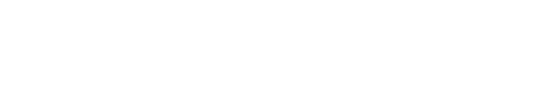



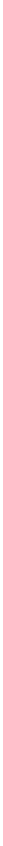

Figure6

Figure6

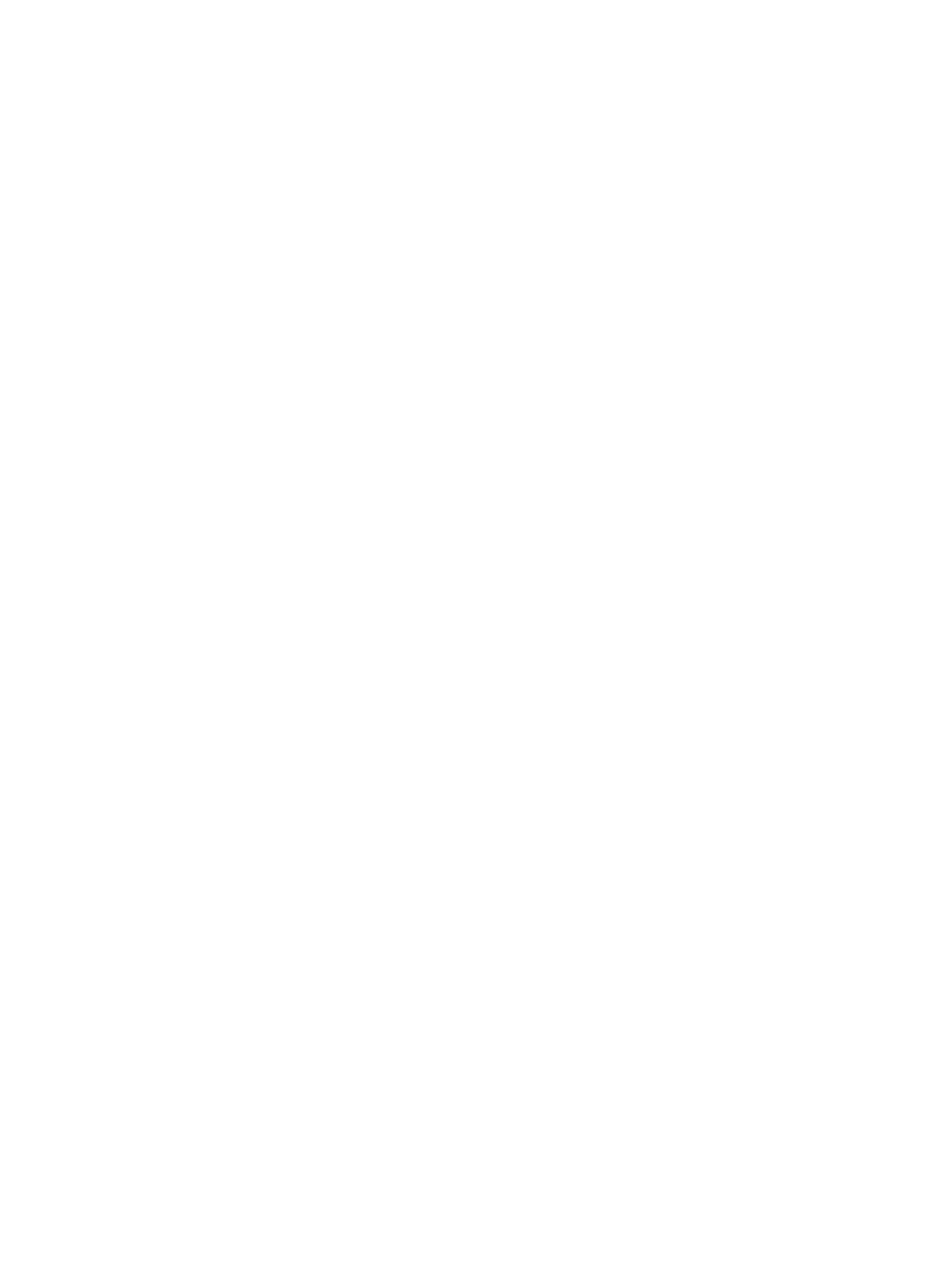




\begin{tabular}{|c|c|c|c|}
\hline $\begin{array}{c}\text { Coil } \\
\text { family }\end{array}$ & $\begin{array}{c}\text { DHVT at 4K } \\
\text { OK }\end{array}$ & $\begin{array}{c}\text { DHVT at 4K NOT } \\
\text { OK }\end{array}$ & \% of success \\
\hline NPCs & 19 & 21 & 47.5 \\
\hline PCs & 12 & 10 & 54.5 \\
\hline
\end{tabular}

Table 2: Summary of all the DHVT at cryogenic temperature only. The reported numbers represent the number of tested coils. 

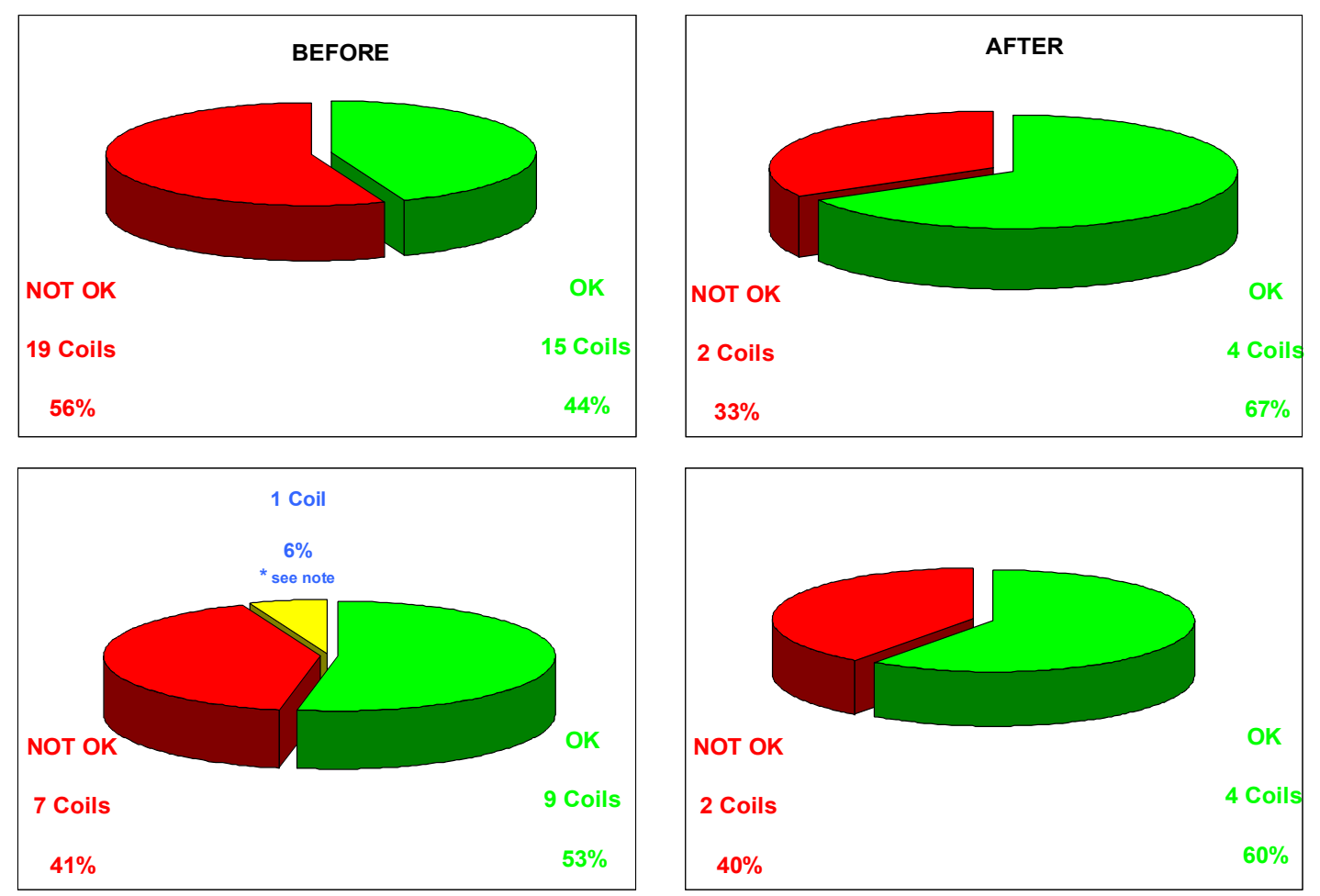

Graph 1: For NPCs the successful DC tests increased from $44 \%$ to $67 \%$; similarly for PCs the success rate passed from $53 \%$ to $60 \%$.

$\begin{aligned} *= & \text { one only planar coil at cold condition gave failed unlike at warm condition. }\end{aligned}$

C


